\title{
A Convenient One-Pot Biginelli Reaction Catalyzed by Y(OAc) 3 : An Improved Protocol for the Synthesis of 3,4-Dihydropyrimidin-2(1H)-ones and Their Sulfur Analogues
}

\author{
Gopalakrishnan Aridoss and Yeon Tae Jeong* \\ Division of Image Science and Information Engineering, Pukyong National University, Busan 608 739, Korea \\ *E-mail: ytjeong@pknu.ac.kr \\ Received December 2, 2009, Accepted February 3, 2010
}

\begin{abstract}
Yttrium(III) acetate hydrate-catalyzed novel synthesis of 3,4-dihydropyrimidin-2(1H)-(thio)one derivatives was achieved through one-pot three-component condensation of diversified aldehydes, $\beta$-ketoesters and urea or $N$-methylurea or thiourea with a molar ratio of 1:1:1.4. In comparison to the classical Biginelli approach, this catalytic method has the advantages of short reaction time and improved product yield.
\end{abstract}

Key Words: Biginelli reaction, 3,4-Dihydropyrimidin-2(1H)-one, Yttrium acetate

\section{Introduction}

Multicomponent reactions (MCRs) ${ }^{1-3}$ are emerged as a well established technique for the facile construction of complex and structurally different target molecules in one-pot fashion from at least three diverse simple building blocks. Owing to the distinctive synthetic features like selectivity, atom-economy and convergence, MCR offers remarkable advantages over conventional linear multi-step synthesis and consequently gains increasing importance in the field of organic and medicinal chemistry. Diversely functionalized 3,4-dihydropyrimidin-2(1H)-ones (DHPMs) synthesized by way of the one-pot Biginelli threecomponent condensation have attracted considerable interest in industry as well as in academia because of their wide scope of promising pharmacological activities, such as calcium channel blockers, antihypertensive, antifungal, antiviral, antibacterial, anti-inflammatory, analgesic and anticancer drugs. Hence, they are classified as one of the most important groups of drug like scaffold. ${ }^{4-7}$ A recent emphasis in this perspective has been the identification of the structurally simple DHPM derivative viz., monastrol as a mitotic kinesin Eg5 motor protein inhibitor for the development of anticancer drugs. ${ }^{8}$ The great potential of DHPMs in biological and pharmaceutical fields has accordingly triggered growing interest in their synthetic study.

Even though the most simple and straightforward practice to synthesize DHPMs is the century-old Biginelli's reaction ${ }^{9}$ involving mineral acid-catalyzed three-component condensation of aldehyde, easily enolizable carbonyl compound and urea, it often provides only low to moderate yields of the desired target molecules when substituted aromatic aldehydes or thioureas are employed and also requires longer reaction time. ${ }^{4,10-14}$ Therefore, a plethora of improved synthetic procedures based on the modifications of traditional Biginelli condensation have been developed during the past few years which instead of traditional protic acids, such as $\mathrm{HCl}_{1}{ }^{9}$ con. $\mathrm{H}_{2} \mathrm{SO}_{4}{ }^{14,15}$ and silica $\mathrm{H}_{2} \mathrm{SO}_{4}{ }^{16}$ involves the use of a variety of Lewis acid catalysts, such as $\mathrm{BF}_{3}$ $\mathrm{OEt}_{2} / \mathrm{CuCl} / \mathrm{HOAc},{ }^{10} \mathrm{NiCl}_{2} \cdot 6 \mathrm{H}_{2} \mathrm{O} / \mathrm{HCl}^{11}, \mathrm{InBr}_{3},{ }^{17} \mathrm{Mn}(\mathrm{OAc})_{3}$. $2 \mathrm{H}_{2} \mathrm{O},{ }^{18} \mathrm{Fe}\left(\mathrm{CF}_{3} \mathrm{CO}_{2}\right)_{3},{ }^{19} \mathrm{Fe}\left(\mathrm{CF}_{3} \mathrm{SO}_{3}\right)_{3},{ }^{19} \mathrm{SiCl}_{4},{ }^{20} \mathrm{VCl}_{3},{ }^{21} \mathrm{Y}\left(\mathrm{NO}_{3}\right)_{3}$.
$6 \mathrm{H}_{2} \mathrm{O},{ }^{21 \mathrm{a}}$ Yttria-zirconia ${ }^{21 \mathrm{~b}}$ and $p$-TsOH ${ }^{22}$ etc. Nevertheless, in spite of their potential utility, some of the reported methods involve longer reaction time, unsatisfactory yield, incompatibility with wide range of functional groups, catalyst which needs dry condition and difficult product isolation procedures. Although yttrium nitrate ${ }^{21 a}$ requires minimum reaction time with reasonable yield, the methodology has not been extended well to the synthesis of biologically important diversified $\mathrm{N}$-methyl and thio derivatives of DHPMs. ${ }^{4,5}$ Consequently, development of efficient catalyst for the synthesis of this multifunctionalized DHPMs with variations of substituents in all three components besides improvement in yield would be desirable. The literature survey indicates that $\mathrm{Y}(\mathrm{OAc})_{3}$ hydrate has not been reported as Lewis acid catalysts for Biginelli reaction.

Therefore, in continuation of our recent work on the construction of biologically potent heterocyclic frameworks, ${ }^{23,24}$ we describe in this paper a simple and improved approach for the generation of library of 3,4-dihydropyrimidin-2(1H)-ones and their sulfur analogues through Biginelli's three component condensation by using yttrium(III) acetate hydrate as a new Lewis acid catalyst preserving the simplicity of the classical one-pot protocol (Scheme 1).

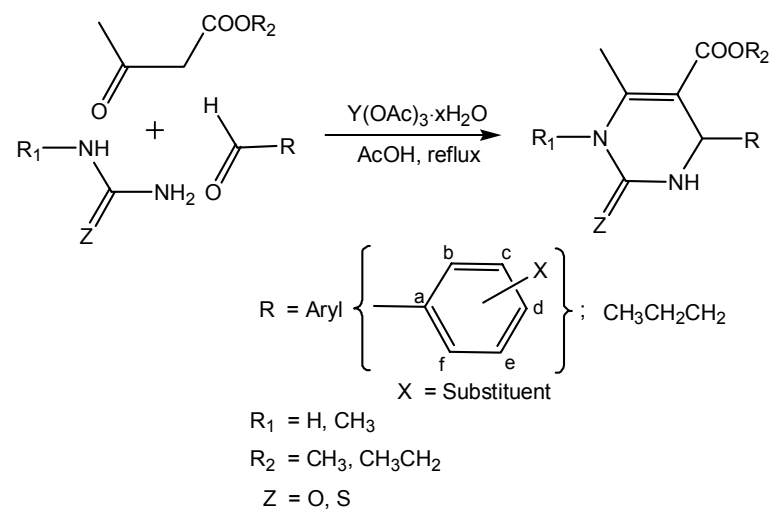

Scheme 1. General synthetic pathway for the synthesis of compounds $1-46$ 


\section{Experimental Part}

Yttrium(III) acetate hydrate (product code no. 326046 99.9\%) and all other reagents were procured from Sigma Aldrich and used as received. The course of the reactions and purity of the products were assessed by performing TLC. Melting points were read using Electrothermal-9100 (Japan) instrument and are uncorrected. NMR spectra were run on JEOL (Japan) JNM ECP-400 instrument operating at $400 \mathrm{MHz}$ for ${ }^{1} \mathrm{H}$ and 100.6 $\mathrm{MHz}$ for completely proton decoupled ${ }^{13} \mathrm{C}$ using $\mathrm{CDCl}_{3}$ or DMSO- $d_{6}$ as solvent and tetramethylsilane (TMS) as internal standard. Mass spectra were taken on a JEOL, JMS-700 instrument. Data are reported as follows: chemical shift (multiplicity [singlet (s), doublet (d), dd (double doublet), triplet (t), quartet (q), broad (br), and multiplet (m)], integration, coupling constants [quoted to the nearest $0.1 \mathrm{~Hz}$ ] and assignment).

General procedure for the synthesis of $N$-unsubstituted and/or $\mathrm{N}$-methyl substituted 3,4-dihydropyrimidin-2(1H)-(thio)ones (1-46). A solution of aldehyde ( $5 \mathrm{mmol}), \beta$-keto ester $(5 \mathrm{mmol})$ and urea or $N$-methylurea or thiourea $(7 \mathrm{mmol})$ in acetic acid $(3 \mathrm{~mL})$ was heated at $115^{\circ} \mathrm{C}$ in the presence of yttrium(III) acetate hydrate $(0.4 \mathrm{mmol}-8 \mathrm{~mol} \%)$ for a specified time (as mentioned in Table 1). The reaction mixture (after being cooled to room temperature) was poured onto crushed ice and quenched with $10 \% \mathrm{NaHCO}_{3}$ solution. The solid separated after stirring for 15 - 20 min was filtered under suction, washed with ice-cold water $(100 \mathrm{~mL})$, ethanol-water mixture $(7+13 \mathrm{~mL})$ and dried. Pure compound was obtained by recrystallization in excess of hot ethanol or in aqueous ethanol.

All the known products in the literature were identified by comparing their analytical and spectral data while the new compounds (given below) were characterized by their melting point, NMR and mass spectra. The results are summarized in Table 1.

5-Ethoxycarbonyl-1,6-dimethyl-4-(2-bromophenyl)-3,4-dihydropyrimidin-2(1H)-one (16): Solid, mp 142 - $143{ }^{\circ} \mathrm{C} ;{ }^{1} \mathrm{H}$ NMR (400 MHz, DMSO- $\left.d_{6}\right) \delta 7.90(\mathrm{~d}, 1 \mathrm{H}, J=3.29, \mathrm{H}-3), 7.58$ (d, $1 \mathrm{H}, J=8.06, \mathrm{H}-\mathrm{c}), 7.36-7.29$ (m, 2H, H-d and H-f), 7.21-7.17 (m, 1H, H-e), 5.60 (d, 1H, J=3.66, H-4), 3.97-3.88 (m, 2H, $\mathrm{CH}_{2}$ of ester), $3.15\left(\mathrm{~s}, 3 \mathrm{H}, \mathrm{N}-\mathrm{CH}_{3}\right), 2.55\left(\mathrm{~s}, 3 \mathrm{H}, \mathrm{CH}_{3}\right.$ at $\left.\mathrm{C}-6\right), 1.01(\mathrm{t}$, $3 \mathrm{H}, J=7.14, \mathrm{CH}_{3}$ of ester); ${ }^{13} \mathrm{C} \mathrm{NMR}\left(100 \mathrm{MHz}, \mathrm{DMSO}-d_{6}\right) \delta$ 165.1, 152.0, 151.3, 142.5, 132.6, 129.5, 128.5, 128.4, 122.3, 101.3, 59.3, 52.7, 29.6, 15.9, 13.9; MS m/z, (\%) $354\left[(\mathrm{M}+2)^{+}\right.$, 5.18], $352\left(\mathrm{M}^{+}, 4.84\right), 323$ (12.8), 279 (17.23), 273 (43.34), 243 (6.83), 197 (100), 169 (40.06), 151 (31.28), 115 (6.79), 56 (65.16).

5-Ethoxycarbonyl-1,6-dimethyl-4-(4-bromophenyl)-3,4-dihydropyrimidin-2(1H)-one (17): Solid, mp 150 - $151{ }^{\circ} \mathrm{C} ;{ }^{1} \mathrm{H}$ NMR (400 MHz, DMSO- $\left.d_{6}\right) \delta 8.03(\mathrm{~d}, 1 \mathrm{H}, J=3.66, \mathrm{H}-3), 7.53$ (d, 2H, $J=8.42$, H-c and H-e), 7.19 (d, 2H, $J=8.06, \mathrm{H}-\mathrm{b}$ and $\mathrm{H}-\mathrm{f}$ ), 5.16 (d, 1H, $J=3.66$, H-4), 4.04 (q, $2 \mathrm{H}, J=6.96, J=14.28$, $\mathrm{CH}_{2}$ of ester), $3.11\left(\mathrm{~s}, 3 \mathrm{H}, \mathrm{N}-\mathrm{CH}_{3}\right), 2.50$ (s, $3 \mathrm{H}, \mathrm{CH}_{3}$ at C-6), 1.12 (t, $3 \mathrm{H}, J=7.14, \mathrm{CH}_{3}$ of ester); ${ }^{13} \mathrm{C}$ NMR (100 MHz, DMSO- $\left.d_{6}\right)$ $\delta 165.4,152.8,150.9,143.4,131.3,128.3,120.3,101.9,59.5$, 51.9, 29.7, 15.9, 13.9; MS $m / z,(\%) 354\left[(\mathrm{M}+2)^{+}, 6.37\right], 352$ $\left(\mathrm{M}^{+}, 7.22\right), 339(7.40), 323(31.27), 279$ (18.59), $197(100), 169$ (40.86), 151 (35.20), 115 (6.87), 66 (7.21), 56 (61.29); Anal. Calcd for $\mathrm{C}_{15} \mathrm{H}_{17} \mathrm{BrN}_{2} \mathrm{O}_{3}$ : C, 51.01; H, 4.85; N 7.93. Found: $\mathrm{C}$, $50.81 ; \mathrm{H}, 4.72 ; \mathrm{N}, 7.85$.
5-Ethoxycarbonyl-1,6-dimethyl-4-(2-methoxyphenyl)-3,4dihydropyrimidin-2(1H)-one (18): Solid, $\operatorname{mp~} 145-146{ }^{\circ} \mathrm{C} ;{ }^{1} \mathrm{H}$ NMR (400 MHz, DMSO-d $) \delta 7.52$ (d, 1H, $J=3.29, \mathrm{H}-3), 7.25-$ 7.21 (m, 1H, $J=8.42$, H-d), 7.04 (dd, $1 \mathrm{H}, J=1.65, J=7.51$, H-f), 6.96 (d, 1H, $J=8.06, \mathrm{H}-\mathrm{e}), 6.86(\mathrm{t}, 1 \mathrm{H}, J=7.32, \mathrm{H}-\mathrm{c}), 5.46$ (d, $1 \mathrm{H}, J=3.29$, H-4), 3.99-3.94 (m, $2 \mathrm{H}, \mathrm{CH}_{2}$ of ester), 3.77 (s, $3 \mathrm{H}$, $\left.\mathrm{O}-\mathrm{CH}_{3}\right), 3.10\left(\mathrm{~s}, 3 \mathrm{H}, \mathrm{N}-\mathrm{CH}_{3}\right), 2.49\left(\mathrm{~s}, 3 \mathrm{H}, \mathrm{CH}_{3}\right.$ at C-6), 1.05 (t, $3 \mathrm{H}, J=7.14, \mathrm{CH}_{3}$ of ester); ${ }^{13} \mathrm{C}$ NMR (100 MHz, DMSO- $\left.d_{6}\right) \delta$ 165.6, 156.6, 153.1, 150.4, 130.8, 128.7, 126.9, 120.1, 111.1, 101.1, 59.3, 55.4, 47.9, 29.6, 15.9, 13.9; MS m/z, (\%) $304\left[\mathrm{M}^{+}\right.$, 26.41], 289 (21.72), 275 (100), 257 (15.57), 232 (10.66), 231 (64.70), 216 (15.22), 197 (86.17), 169 (50.09), 151 (45.67), 115 (11.74), 91 (10.26), 77 (16.86), 56 (93.19).

5-Ethoxycarbonyl-6-methyl-4-(2-bromophenyl)-3,4-dihydropyrimidin-2(1H)-thione (22): Solid, mp $149-150{ }^{\circ} \mathrm{C} ;{ }^{1} \mathrm{H}$ NMR (400 MHz, DMSO- $\left.d_{6}\right) \delta 10.36$ (s, $\left.1 \mathrm{H}, \mathrm{H}-1\right), 9.60$ (s, $1 \mathrm{H}$, H-3), 7.59 (d, 1H, J=8.06, H-c), 7.39 (t, 1H, J=7.39, H-e), 7.29 (d, 1H, $J=6.59$, H-d), 7.23-7.19 (m, 1H, H-f), 5.62 (d, 1H, $J=$ 2.93, H-4), 3.92 (q, 2H, $J=6.96, J=14.28, \mathrm{CH}_{2}$ of ester), 2.33 (s, $3 \mathrm{H}, \mathrm{CH}_{3}$ at $\left.\mathrm{C}-6\right), 1.01$ (t, $3 \mathrm{H}, J=7.14, \mathrm{CH}_{3}$ of ester); ${ }^{13} \mathrm{C} \mathrm{NMR}$ $\left(100 \mathrm{MHz}, \mathrm{DMSO}-d_{6}\right) \delta 173.8,164.7,145.4,142.3,132.7$, $129.7,129.3,128.4,122.2,100.1,59.4,54.1,16.9,13.8 ; \mathrm{MS}$ $m / z,(\%) 356\left[(\mathrm{M}+2)^{+}, 36.55\right], 354\left(\mathrm{M}^{+}, 33.63\right), 327(23.34), 275$ (50.28), 199 (100), 171 (26.57), 153 (13.67), 126 (9.03), 102 (15.53), 67 (10.56), 51(6.12).

5-Ethoxycarbonyl-6-methyl-4-(4-bromophenyl)-3,4-dihydropyrimidin-2(1H)-thione (23): Solid, mp $182-183{ }^{\circ} \mathrm{C} ;{ }^{1} \mathrm{H}$ NMR (400 MHz, DMSO- $\left.d_{6}\right) \delta 10.38$ (s, 1H, H-1), 9.66 (s, 1H, $\mathrm{H}-3$ ), 7.56 (d, 2H, $J=8.42$, H-c and H-e), 7.17 (d, 2H, $J=8.42$, $\mathrm{H}-\mathrm{b}$ and $\mathrm{H}-\mathrm{f}$ ), 5.15 (d, 1H, $J=3.66, \mathrm{H}-4), 4.00$ (q, 2H, $J=6.96$, $J=13.51, \mathrm{CH}_{2}$ of ester), $2.29\left(\mathrm{~s}, 3 \mathrm{H}, \mathrm{CH}_{3}\right.$ at C-6), 1.09 (t, $3 \mathrm{H}$, $J=7.14, \mathrm{CH}_{3}$ of ester); ${ }^{13} \mathrm{C}$ NMR $\left(100 \mathrm{MHz}\right.$, DMSO- $\left.d_{6}\right) \delta 174.2$, 164.9, 145.3, 142.7, 131.4, 128.6, 120.7, 100.2, 59.6, 53.5, 17.1, 13.9; MS m/z, (\%) $356\left[(\mathrm{M}+2)^{+}, 34.91\right], 354\left(\mathrm{M}^{+}, 32.20\right), 327$ (52.12), 281 (34.22), 199 (100), 171 (33.56), 153 (18.49), 126 (14.43), 102 (15.56), 67 (13.54), 51 (7.07); Anal. Calcd for $\mathrm{C}_{14-}$ $\mathrm{H}_{15} \mathrm{BrN}_{2} \mathrm{O}_{2} \mathrm{~S}$ : C, 47.33; H, 4.26; N 7.89. Found: C, 47.56; H, 4.14; N, 7.96.

5-Methoxycarbonyl-6-methyl-4-(2-bromophenyl)-3,4-dihydropyrimidin-2(1H)-one (28): Solid, mp $240-242{ }^{\circ} \mathrm{C}$; ${ }^{1} \mathrm{H}$ NMR (400 MHz, DMSO- $\left.d_{6}\right) \delta 9.31$ (s, 1H, H-1), 7.71 (s, 1H, H-3), 7.57 (d, 1H, $J=7.69$, H-c), 7.37 (t, 1H, $J=7.14, \mathrm{H}-\mathrm{e}), 7.31$ (dd, $1 \mathrm{H}, J=1.83, J=7.69, \mathrm{H}-\mathrm{d}), 7.21-7.17$ (m, 1H, H-f), 5.59 (d, 1H, $J=2.93, \mathrm{H}-4), 3.45$ (s, 3H, $\mathrm{CH}_{3}$ of ester), 2.29 (s, 3H, $\mathrm{CH}_{3}$ at C-6); ${ }^{13} \mathrm{C}$ NMR (100 MHz, DMSO- $\left.d_{6}\right) \delta 165.4,151.2,149.3$, 143.2, 132.6, 129.3, 128.6, 128.4, 122.1, 98.1, 53.9, 50.6, 17.7; MS $m / z,(\%) 326\left[(\mathrm{M}+2)^{+}, 2.79\right], 324\left(\mathrm{M}^{+}, 2.81\right), 265$ (18.44), 245 (75.29), 169 (100), 137 (49.63), 115 (14.88), 102 (15.37), 89 (8.14), 75 (17.37), 50 (9.47).

5-Methoxycarbonyl-6-methyl-4-(4-bromophenyl)-3,4-dihydropyrimidin-2(1H)-one (29): Solid, mp $218-220{ }^{\circ} \mathrm{C} ;{ }^{1} \mathrm{H}$ NMR $\left(400 \mathrm{MHz}, \mathrm{DMSO}-d_{6}\right) \delta 9.28$ (s, 1H, H-1), 7.79 (s, 1H, H-3), 7.53 (d, 2H, $J=8.42$, H-c and H-e), 7.18 (d, 2H, $J=8.42, \mathrm{H}-\mathrm{b}$ and $\mathrm{H}-\mathrm{f}), 5.12$ (d, $1 \mathrm{H}, J=3.29, \mathrm{H}-4), 3.53$ (s, 3H, $\mathrm{CH}_{3}$ of ester), 2.25 (s, $3 \mathrm{H}, \mathrm{CH}_{3}$ at C-6); ${ }^{13} \mathrm{C}$ NMR (100 MHz, DMSO- $\left.d_{6}\right) \delta 165.7$, 151.9, 148.9, 143.9, 131.3, 128.4, 120.3, 98.5, 53.3, 50.8, 17.8; MS $m / z,(\%) 326\left[(\mathrm{M}+2)^{+}, 6.00\right], 324\left(\mathrm{M}^{+}, 7.09\right), 309$ (17.79), 265 (18.04), 245 (11.99), 169 (100), 137 (49.46), 110 (11.90), 
102 (8.52), 75 (13.13), 50 (7.81); Anal. Calcd for $\mathrm{C}_{13} \mathrm{H}_{13} \mathrm{BrN}_{2} \mathrm{O}_{3}$ : C, 48.02; H, 4.03; N 8.62. Found: C, 48.21; H, 4.12; N, 7.50.

5-Methoxycarbonyl-6-methyl-4-(propyl)-3,4-dihydropyrimidin-2(1H)-one (34): Solid, mp $151-152{ }^{\circ} \mathrm{C} ;{ }^{1} \mathrm{H}$ NMR (400 MHz, DMSO- $\left.d_{6}\right) \delta 8.97$ (s, 1H, H-1), 7.35 (s, 1H, H-3), 4.064.02 (m, 1H, H-4), 3.59 (s, 3H, $\mathrm{CH}_{3}$ of ester), 2.15 (s, 3H, $\mathrm{CH}_{3}$ at C-6), 1.37-1.15 [m, 4H, $\left(\mathrm{CH}_{2}\right)_{2}$ of propyl], 0.83 (t, $3 \mathrm{H}, J=6.77$, $\mathrm{CH}_{3}$ of propyl); ${ }^{13} \mathrm{C} \mathrm{NMR}\left(100 \mathrm{MHz}, \mathrm{DMSO}-d_{6}\right) \delta 166.0,152.9$, 148.6, 99.3, 50.8, 49.9, 17.8, 17.1, 13.9; $\mathrm{MS} \mathrm{m} / z$, (\%) 212 [(M) ${ }^{+}$, 0.47], 181 (4.88), 169 (100), 137 (63), 125 (9.26), 110 (7.93), 96 (8.04), 67 (6.49).

5-Methoxycarbonyl-1,6-dimethyl-4-(2-bromophenyl)-3,4dihydropyrimidin-2(1H)-one (36): Solid, mp $164-165{ }^{\circ} \mathrm{C} ;{ }^{1} \mathrm{H}$ NMR (400 MHz, DMSO- $\left.d_{6}\right) \delta 7.90$ (d, $\left.1 \mathrm{H}, J=3.66, \mathrm{H}-3\right), 7.58$ (d, 1H, J=8.06, H-c), 7.36-7.29 (m, 2H, H-f and H-e), 7.21-7.17 $(\mathrm{m}, 1 \mathrm{H}, \mathrm{H}-\mathrm{d}), 5.58(\mathrm{~d}, 1 \mathrm{H}, J=3.66, \mathrm{H}-4), 3.48$ (s, 3H, $\mathrm{CH}_{3}$ of ester), $3.15\left(\mathrm{~s}, 3 \mathrm{H}, \mathrm{N}-\mathrm{CH}_{3}\right), 2.55\left(\mathrm{~s}, 3 \mathrm{H}, \mathrm{CH}_{3}\right.$ at $\left.\mathrm{C}-6\right) ;{ }^{13} \mathrm{C} \mathrm{NMR}(100$ MHz, DMSO- $\left.d_{6}\right) \delta 165.7,152.1,151.5,142.4,132.7,129.5$, $128.5,128.4,122.2,101.1,52.5,50.9,29.6,16.0 ; \mathrm{MS} m / z,(\%)$ $340\left[(\mathrm{M}+2)^{+}, 7.80\right], 338\left(\mathrm{M}^{+}, 8.12\right), 323$ (22.38), 279 (14.84), 183 (100), 151 (50.71), 94 (7.14), 66 (5.85), 56 (40.35).

5-Methoxycarbonyl-1,6-dimethyl-4-(4-bromophenyl)-3,4dihydropyrimidin-2(1 H)-one (37): Solid, mp $161-162{ }^{\circ} \mathrm{C} ;{ }^{1} \mathrm{H}$ NMR (400 MHz, DMSO- $\left.d_{6}\right) \delta 8.04$ (d, $1 \mathrm{H}, J=4.03, \mathrm{H}-1$ ), 7.52 (d, 2H, $J=8.42$, H-c and H-e), 7.18 (d, 2H, $J=8.42, \mathrm{H}-\mathrm{b}$ and $\mathrm{H}-\mathrm{f}), 5.15$ (d, 1H, $J=3.66, \mathrm{H}-4), 3.58$ (s, 3H, $\mathrm{CH}_{3}$ of ester), 3.09 $\left(\mathrm{s}, 3 \mathrm{H}, \mathrm{N}-\mathrm{CH}_{3}\right), 2.50\left(\mathrm{~s}, 3 \mathrm{H}, \mathrm{CH}_{3}\right.$ at $\left.\mathrm{C}-6\right) ;{ }^{13} \mathrm{C} \mathrm{NMR}(100 \mathrm{MHz}$, DMSO- $\left.d_{6}\right) \delta 165.9,152.9,151.2,143.2,131.4,128.3,120.4$, 101.6, 51.7, 51.1, 29.7, 16.1; MS $m / z,(\%) 340\left[(\mathrm{M}+2)^{+}, 6.60\right]$, $338\left(\mathrm{M}^{+}, 6.86\right), 323$ (20.72), 279 (14.04), 183 (100), 151 (47.33), 115 (5.01), 94 (7.94), 75 (6.34), 56 (44.42).

5-Methoxycarbonyl-1,6-dimethyl-4-(2-methoxyphenyl)3,4-dihydropyrimidin-2(1H)-one (38): Solid, mp $170-172{ }^{\circ} \mathrm{C}$; ${ }^{1} \mathrm{H}$ NMR (400 MHz, DMSO- $d_{6}$ ) $\delta 7.50$ (d, $1 \mathrm{H}, J=3.66, \mathrm{H}-3$ ), 7.24 (t, 1H, $J=7.14, \mathrm{H}-\mathrm{d}), 7.02$ (d, 1H, $J=6.59$, H-f), 6.99 (d, $1 \mathrm{H}, J=8.42$, H-e), 6.86 (t, 1H, $J=7.32$, H-c), 5.45 (d, 1H, $J=$ 3.66, H-4), 3.78 (s, 3H, O-CH $\mathrm{CH}_{3}, 3.51$ (s, $3 \mathrm{H}, \mathrm{CH}_{3}$ of ester), 3.09 $\left(\mathrm{s}, 3 \mathrm{H}, \mathrm{N}-\mathrm{CH}_{3}\right), 2.50\left(\mathrm{~s}, 3 \mathrm{H}, \mathrm{CH}_{3}\right.$ at $\left.\mathrm{C}-6\right) ;{ }^{13} \mathrm{C} \mathrm{NMR}(100 \mathrm{MHz}$, DMSO- $\left.d_{6}\right) \delta 166.1,156.6,153.2,150.7,130.4,128.7,126.5$, 120.1, 111.2, 100.6, 55.4, 50.9, 47.8, 29.6, 15.9; MS m/z, (\%) $290\left(\mathrm{M}^{+}, 26.08\right), 275$ (69.92), 259 (14.27), 231 (57.78), 216 (19.54), 199 (17.66), 183 (100), 151 (66.84), 115 (12.15), 94 (15.63), 91 (10.64), 77 (18.85), 56 (96.55).

5-Methoxycarbonyl-1,6-dimethyl-4-(4-nitrophenyl)-3,4dihydropyrimidin-2(1H)-one (40): Solid, mp $176-178{ }^{\circ} \mathrm{C} ;{ }^{1} \mathrm{H}$ NMR $\left(400 \mathrm{MHz}, \mathrm{DMSO}-d_{6}\right) \delta 8.19(\mathrm{~d}, 2 \mathrm{H}, J=8.79, \mathrm{H}-\mathrm{c}$ and H-e), 8.17 (d, 1H, $J=4.03, \mathrm{H}-1), 7.49$ (d, 2H, $J=8.79$, H-b and $\mathrm{H}-\mathrm{f}), 5.28(\mathrm{~d}, 1 \mathrm{H}, J=3.66, \mathrm{H}-4), 3.58$ (s, $3 \mathrm{H}, \mathrm{CH}_{3}$ of ester), 3.10 $\left(\mathrm{s}, 3 \mathrm{H}, \mathrm{N}-\mathrm{CH}_{3}\right), 2.5\left(\mathrm{~s}, 3 \mathrm{H}, \mathrm{CH}_{3}\right.$ at $\left.\mathrm{C}-6\right) ;{ }^{13} \mathrm{C} \mathrm{NMR}(100 \mathrm{MHz}$, DMSO- $\left.d_{6}\right) \delta 165.7,152.7,151.9,151.0,146.7,127.4,123.7$, 101.0, 51.9, 51.1, 29.8, 16.1; MS m/z, (\%) $305\left(\mathrm{M}^{+}, 8.98\right), 290$ (28.99), 246 (11.85), 200 (17.50), 183 (100), 151 (69.90), 115 (9.12), 94 (12.76), 66 (10.92), 56 (59.63).

5-Methoxycarbonyl-6-methyl-4-(2-bromophenyl)-3,4-dihydropyrimidin-2(1H)-thione (42): Solid, mp $172-173{ }^{\circ} \mathrm{C} ;{ }^{1} \mathrm{H}$ NMR (400 MHz, DMSO- $\left.d_{6}\right) \delta 10.39$ (s, 1H, H-1), 9.61 (s, 1H, H-3), 7.59 (d, 1H, J=7.69, H-c), 7.39 (t, 1H, J=7.49, H-e), 7.29 (dd, $1 \mathrm{H}, J=1.47, J=7.69$, H-d), 7.23-7.19 (m, 1H, H-f), 5.61 (d,
$1 \mathrm{H}, J=3.29, \mathrm{H}-4), 3.48$ (s, 3H, $\mathrm{CH}_{3}$ of ester), 2.32 (s, 3H, $\mathrm{CH}_{3}$ at C-6); ${ }^{13} \mathrm{C}$ NMR (100 MHz, DMSO- $\left.d_{6}\right) \delta 173.9,165.2,145.5$, 142.3, 132.8, 129.7, 129.2, 128.5, 122.1, 99.9, 53.9, 50.9, 16.9; MS $m / z,(\%) 342\left[(\mathrm{M}+2)^{+}, 26.37\right], 340\left(\mathrm{M}^{+}, 26.54\right), 327$ (7.53), 281 (16.14), 261 (37), 185 (100), 153 (25.70), 143 (8.68), 126 (13.88), 102 (12.93), 75 (9.25), 59 (7.05).

5-Methoxycarbonyl-6-methyl-4-(4-bromophenyl)-3,4-dihydropyrimidin-2(1H)-thione (43): Solid, mp $153-154{ }^{\circ} \mathrm{C} ;{ }^{1} \mathrm{H}$ NMR (400 MHz, DMSO- $\left.d_{6}\right) \delta 10.40$ (s, 1H, H-1), 9.68 (d, 1H, $J=2.93, \mathrm{H}-3), 7.55$ (d, 2H, $J=8.42$, H-c and H-e), 7.16 (d, 2H, $J=8.42, \mathrm{H}-\mathrm{b}$ and $\mathrm{H}-\mathrm{f}), 5.15$ (d, $1 \mathrm{H}, J=3.66, \mathrm{H}-4), 3.55$ (s, 3H, $\mathrm{CH}_{3}$ of ester), 2.29 (s, 3H, $\mathrm{CH}_{3}$ at C-6); ${ }^{13} \mathrm{C} \mathrm{NMR}(100 \mathrm{MHz}$, DMSO- $\left.d_{6}\right) \delta 174.3,165.5,145.5,142.5,131.5,128.6,120.8$, 99.9, 53.3, 51.1, 17.2; MS m/z, (\%) $342\left[(\mathrm{M}+2)^{+}, 27.17\right], 340$ $\left(\mathrm{M}^{+}, 28.46\right), 327$ (20.59), 281 (19.29), 261 (6.06), 185 (100), 153 (23.61), 143 (8.44), 126 (14.13), 102 (10.92), 67 (9.80), 59 (5.82).

5-Methoxycarbonyl-6-methyl-4-(4-hydroxyphenyl)-3,4-dihydropyrimidin-2(1H)-thione (46): Solid, $\mathrm{mp} 245-246{ }^{\circ} \mathrm{C} ;{ }^{1} \mathrm{H}$ NMR (400 MHz, DMSO- $\left.d_{6}\right) \delta 10.26(\mathrm{~s}, 1 \mathrm{H}, \mathrm{H}-1), 9.56(\mathrm{~d}, 1 \mathrm{H}$, $J=1.83, \mathrm{H}-3), 9.45$ (bs, $1 \mathrm{H}, \mathrm{Ph}-\mathrm{OH}), 7.01$ (d, 2H, $J=8.79, \mathrm{H}-\mathrm{b}$ and $\mathrm{H}-\mathrm{f}), 6.71$ (d, 2H, $J=8.42$, H-c and H-e), 5.06 (d, 1H, $J=$ 3.66, $\mathrm{H}-4), 3.54$ (s, 3H, $\mathrm{CH}_{3}$ of ester), 2.28 (s, 3H, $\mathrm{CH}_{3}$ at $\left.\mathrm{C}-6\right)$; ${ }^{13} \mathrm{C}$ NMR (100 MHz, DMSO- $\left.d_{6}\right) \delta 173.9,165.7,156.9,144.7$, $133.9,127.5,115.2,100.9,53.4,50.9,17.1 ; \mathrm{MS} m / z,(\%) 278$ $\left(\mathrm{M}^{+}, 78.83\right), 263$ (57.89), 245 (11.76), 219 (94.52), 217 (9.97), 185 (100), 153 (29.83), 145 (22.54), 126 (27.64), 115 (24.53), 94 (11.67), 89 (30.18), 65 (34.37), 59 (23.94).

\section{Results and Discussion}

In order to assess the catalytic activity of yttrium(III) acetate hydrate in Biginelli's one pot condensation, we have carried out a model reaction using benzaldehyde $(5 \mathrm{mmol})$, ethyl acetoacetate $(5 \mathrm{mmol})$, urea $(7.5 \mathrm{mmol})$ and yttrium acetate hydrate $(10$ $\mathrm{mol} \%)$ in refluxing ethanol. Since we got poor yield (28\%) in this model reaction, we have tried the same reaction with different solvents (acetonitrile, THF, DCM, DMSO and DMF) at reflux condition. However, there were no appreciable improvements in these trials also. Then, we tried using 1:1 ratio of each of the said solvents in combination with acetic acid and acetic acid alone as a unique solvent. To our delight, acetic acid alone emerged as an efficient solvent instead of the mixture of solvents in enhancing the yield and also reducing the reaction time (from 18 to $4 \mathrm{~h}$ ) appreciably. For improving the reaction yields, the efficacy of catalyst loading in this reaction was investigated and the optimum amount was found to be $8 \mathrm{~mol} \%$ whereas further increase of the catalyst amount did not increase the yield appreciably. Similarly to optimize the molar ratio of the building blocks, attention was made to carry out reactions with varying amount of the three components. Finally, the cleanest conversions and highest product yields were ideally obtained by using 1:1:1.4 molar ratios of benzaldehyde, ethyl acetoacetate and urea respectively with $8 \mathrm{~mol} \%$ of yttrium acetate hydrate as catalyst.

Having an optimized general protocol for this one pot assembly in hand, we next proceeded with ethyl acetoacetate, urea and a broad range of structurally diverse aromatic aldehydes bearing 
Table 1. Yttrium acetate hydrate-catalyzed synthesis of 3,4-dihydropyrimidin-2(1H)-(thio)ones in one-pot

\begin{tabular}{|c|c|c|c|c|c|c|c|}
\hline DHPM & $\mathrm{R}$ & $\mathrm{R}_{1}$ & $\mathrm{R}_{2}$ & $\mathrm{Z}$ & Yield $^{a}(\%)$ & Time (h) & $\mathrm{Mp}\left({ }^{\circ} \mathrm{C}\right)^{b}$ \\
\hline 1 & $\mathrm{C}_{6} \mathrm{H}_{5}$ & $\mathrm{H}$ & $\mathrm{C}_{2} \mathrm{H}_{5}$ & $\mathrm{O}$ & 92 & 4.0 & $201-202^{10}$ \\
\hline 2 & $4-\mathrm{F}-\mathrm{C}_{6} \mathrm{H}_{4}$ & $\mathrm{H}$ & $\mathrm{C}_{2} \mathrm{H}_{5}$ & $\mathrm{O}$ & 89 & 3.5 & $179-180^{25}$ \\
\hline 3 & $2-\mathrm{Cl}-\mathrm{C}_{6} \mathrm{H}_{4}$ & $\mathrm{H}$ & $\mathrm{C}_{2} \mathrm{H}_{5}$ & $\mathrm{O}$ & 98 & 3 & $218-220^{18}$ \\
\hline 4 & 4-Cl- $\mathrm{C}_{6} \mathrm{H}_{4}$ & $\mathrm{H}$ & $\mathrm{C}_{2} \mathrm{H}_{5}$ & $\mathrm{O}$ & 91 & 4.5 & $215-216^{10}$ \\
\hline 5 & $2-\mathrm{Br}_{-} \mathrm{C}_{6} \mathrm{H}_{4}$ & $\mathrm{H}$ & $\mathrm{C}_{2} \mathrm{H}_{5}$ & $\mathrm{O}$ & 90 & 4 & $207-208^{26}$ \\
\hline 6 & $3-\mathrm{Br}-\mathrm{C}_{6} \mathrm{H}_{4}$ & $\mathrm{H}$ & $\mathrm{C}_{2} \mathrm{H}_{5}$ & $\mathrm{O}$ & 89 & 5.5 & $190-192^{26}$ \\
\hline 7 & $4-\mathrm{Br}^{-} \mathrm{C}_{6} \mathrm{H}_{4}$ & $\mathrm{H}$ & $\mathrm{C}_{2} \mathrm{H}_{5}$ & $\mathrm{O}$ & 87 & 5 & $220-222^{27}$ \\
\hline 8 & $4-\mathrm{HO}-\mathrm{C}_{6} \mathrm{H}_{4}$ & $\mathrm{H}$ & $\mathrm{C}_{2} \mathrm{H}_{5}$ & $\mathrm{O}$ & 92 & 3.5 & $236-237^{17}$ \\
\hline 9 & $4-\mathrm{NO}_{2}-\mathrm{C}_{6} \mathrm{H}_{4}$ & $\mathrm{H}$ & $\mathrm{C}_{2} \mathrm{H}_{5}$ & $\mathrm{O}$ & 92 & 4.5 & $205-207^{10}$ \\
\hline 10 & $2-\mathrm{CH}_{3} \mathrm{O}-\mathrm{C}_{6} \mathrm{H}_{4}$ & $\mathrm{H}$ & $\mathrm{C}_{2} \mathrm{H}_{5}$ & $\mathrm{O}$ & 97 & 0.75 & $260-261^{20}$ \\
\hline 11 & $3-\mathrm{CH}_{3} \mathrm{O}-\mathrm{C}_{6} \mathrm{H}_{4}$ & $\mathrm{H}$ & $\mathrm{C}_{2} \mathrm{H}_{5}$ & $\mathrm{O}$ & 92 & 5 & $214-215^{28}$ \\
\hline 12 & 4- $\mathrm{CH}_{3} \mathrm{O}-\mathrm{C}_{6} \mathrm{H}_{4}$ & $\mathrm{H}$ & $\mathrm{C}_{2} \mathrm{H}_{5}$ & $\mathrm{O}$ & 89 & 4.5 & $200-201^{10}$ \\
\hline 13 & $4-\mathrm{CH}_{3}-\mathrm{C}_{6} \mathrm{H}_{4}$ & $\mathrm{H}$ & $\mathrm{C}_{2} \mathrm{H}_{5}$ & $\mathrm{O}$ & 89 & 3 & $216-217^{17}$ \\
\hline 14 & $\left(\mathrm{CH}_{2}\right)_{2} \mathrm{CH}_{3}$ & $\mathrm{H}$ & $\mathrm{C}_{2} \mathrm{H}_{5}$ & $\mathrm{O}$ & 61 & 6.5 & $156-158^{25}$ \\
\hline 15 & $\mathrm{C}_{6} \mathrm{H}_{5}$ & $\mathrm{CH}_{3}$ & $\mathrm{C}_{2} \mathrm{H}_{5}$ & $\mathrm{O}$ & 84 & 3 & $177^{29}$ \\
\hline 16 & $2-\mathrm{Br}-\mathrm{C}_{6} \mathrm{H}_{4}$ & $\mathrm{CH}_{3}$ & $\mathrm{C}_{2} \mathrm{H}_{5}$ & $\mathrm{O}$ & 94 & 4.5 & $142-143$ \\
\hline 17 & $4-\mathrm{Br}-\mathrm{C}_{6} \mathrm{H}_{4}$ & $\mathrm{CH}_{3}$ & $\mathrm{C}_{2} \mathrm{H}_{5}$ & $\mathrm{O}$ & 93 & 5 & $150-151$ \\
\hline 18 & $2-\mathrm{CH}_{3} \mathrm{O}-\mathrm{C}_{6} \mathrm{H}_{4}$ & $\mathrm{CH}_{3}$ & $\mathrm{C}_{2} \mathrm{H}_{5}$ & $\mathrm{O}$ & 89 & 3.5 & $145-146$ \\
\hline 19 & $4-\mathrm{CH}_{3} \mathrm{O}-\mathrm{C}_{6} \mathrm{H}_{4}$ & $\mathrm{CH}_{3}$ & $\mathrm{C}_{2} \mathrm{H}_{5}$ & $\mathrm{O}$ & 82 & 4 & $134-135^{30}$ \\
\hline 20 & $4-\mathrm{NO}_{2}-\mathrm{C}_{6} \mathrm{H}_{4}$ & $\mathrm{CH}_{3}$ & $\mathrm{C}_{2} \mathrm{H}_{5}$ & $\mathrm{O}$ & 90 & 4 & $107-109^{31}$ \\
\hline 21 & $\mathrm{C}_{6} \mathrm{H}_{5}$ & $\mathrm{H}$ & $\mathrm{C}_{2} \mathrm{H}_{5}$ & $\mathrm{~S}$ & 79 & 4 & $208-209^{27}$ \\
\hline 22 & $2-\mathrm{Br}-\mathrm{C}_{6} \mathrm{H}_{4}$ & $\mathrm{H}$ & $\mathrm{C}_{2} \mathrm{H}_{5}$ & $\mathrm{~S}$ & 82 & 4 & $149-150$ \\
\hline 23 & $4-\mathrm{Br}-\mathrm{C}_{6} \mathrm{H}_{4}$ & $\mathrm{H}$ & $\mathrm{C}_{2} \mathrm{H}_{5}$ & $\mathrm{~S}$ & 82 & 4.5 & $182-183$ \\
\hline 24 & $2-\mathrm{CH}_{3} \mathrm{O}-\mathrm{C}_{6} \mathrm{H}_{4}$ & $\mathrm{H}$ & $\mathrm{C}_{2} \mathrm{H}_{5}$ & $\mathrm{~S}$ & 93 & 3.5 & $188-189^{20}$ \\
\hline 25 & $4-\mathrm{CH}_{3} \mathrm{O}-\mathrm{C}_{6} \mathrm{H}_{4}$ & $\mathrm{H}$ & $\mathrm{C}_{2} \mathrm{H}_{5}$ & $\mathrm{~S}$ & 81 & 4.5 & $152-154^{17}$ \\
\hline 26 & 4-HO- $\mathrm{C}_{6} \mathrm{H}_{4}$ & $\mathrm{H}$ & $\mathrm{C}_{2} \mathrm{H}_{5}$ & $\mathrm{~S}$ & 76 & 3.5 & $199-200^{32}$ \\
\hline 27 & $\mathrm{C}_{6} \mathrm{H}_{5}$ & $\mathrm{H}$ & $\mathrm{CH}_{3}$ & $\mathrm{O}$ & 91 & 4 & $212-214^{10}$ \\
\hline 28 & $2-\mathrm{Br}-\mathrm{C}_{6} \mathrm{H}_{4}$ & $\mathrm{H}$ & $\mathrm{CH}_{3}$ & $\mathrm{O}$ & 95 & 3.5 & $240-242$ \\
\hline 29 & 4-Br- $\mathrm{C}_{6} \mathrm{H}_{4}$ & $\mathrm{H}$ & $\mathrm{CH}_{3}$ & $\mathrm{O}$ & 87 & 3.5 & $218-220$ \\
\hline 30 & $2-\mathrm{CH}_{3} \mathrm{O}-\mathrm{C}_{6} \mathrm{H}_{4}$ & $\mathrm{H}$ & $\mathrm{CH}_{3}$ & $\mathrm{O}$ & 90 & 0.75 & $287-288^{20}$ \\
\hline 31 & $4-\mathrm{CH}_{3} \mathrm{O}-\mathrm{C}_{6} \mathrm{H}_{4}$ & $\mathrm{H}$ & $\mathrm{CH}_{3}$ & $\mathrm{O}$ & 95 & 4.5 & $190-191^{10}$ \\
\hline 32 & 4-HO- $\mathrm{C}_{6} \mathrm{H}_{4}$ & $\mathrm{H}$ & $\mathrm{CH}_{3}$ & $\mathrm{O}$ & 93 & 3.5 & $236-238^{21}$ \\
\hline 33 & $4-\mathrm{NO}_{2}-\mathrm{C}_{6} \mathrm{H}_{4}$ & $\mathrm{H}$ & $\mathrm{CH}_{3}$ & $\mathrm{O}$ & 78 & 4.5 & $231-232^{10}$ \\
\hline 34 & $\left(\mathrm{CH}_{2}\right)_{2} \mathrm{CH}_{3}$ & $\mathrm{H}$ & $\mathrm{CH}_{3}$ & $\mathrm{O}$ & 46 & 6.5 & $151-152$ \\
\hline 35 & $\mathrm{C}_{6} \mathrm{H}_{5}$ & $\mathrm{CH}_{3}$ & $\mathrm{CH}_{3}$ & $\mathrm{O}$ & 86 & 4.5 & $189-190^{29}$ \\
\hline 36 & 2-Br- $\mathrm{C}_{6} \mathrm{H}_{4}$ & $\mathrm{CH}_{3}$ & $\mathrm{CH}_{3}$ & $\mathrm{O}$ & 96 & 4.5 & $164-165$ \\
\hline 37 & 4- $\mathrm{Br}-\mathrm{C}_{6} \mathrm{H}_{4}$ & $\mathrm{CH}_{3}$ & $\mathrm{CH}_{3}$ & $\mathrm{O}$ & 94 & 4.5 & $161-162$ \\
\hline 38 & $2-\mathrm{CH}_{3} \mathrm{O}-\mathrm{C}_{6} \mathrm{H}_{4}$ & $\mathrm{CH}_{3}$ & $\mathrm{CH}_{3}$ & $\mathrm{O}$ & 78 & 4.5 & $170-172$ \\
\hline 39 & $4-\mathrm{CH}_{3} \mathrm{O}-\mathrm{C}_{6} \mathrm{H}_{4}$ & $\mathrm{CH}_{3}$ & $\mathrm{CH}_{3}$ & $\mathrm{O}$ & 76 & 4.5 & $135-137^{29}$ \\
\hline 40 & $4-\mathrm{NO}_{2}-\mathrm{C}_{6} \mathrm{H}_{4}$ & $\mathrm{CH}_{3}$ & $\mathrm{CH}_{3}$ & $\mathrm{O}$ & 80 & 4.5 & $176-178$ \\
\hline 41 & $\mathrm{C}_{6} \mathrm{H}_{5}$ & $\mathrm{H}$ & $\mathrm{CH}_{3}$ & $\mathrm{~S}$ & 80 & 4.5 & $232-234^{20}$ \\
\hline 42 & $2-\mathrm{Br}-\mathrm{C}_{6} \mathrm{H}_{4}$ & $\mathrm{H}$ & $\mathrm{CH}_{3}$ & $\mathrm{~S}$ & 75 & 4.5 & $172-173$ \\
\hline 43 & $4-\mathrm{Br}-\mathrm{C}_{6} \mathrm{H}_{4}$ & $\mathrm{H}$ & $\mathrm{CH}_{3}$ & $\mathrm{~S}$ & 80 & 4.5 & $153-154$ \\
\hline 44 & $2-\mathrm{CH}_{3} \mathrm{O}-\mathrm{C}_{6} \mathrm{H}_{4}$ & $\mathrm{H}$ & $\mathrm{CH}_{3}$ & $\mathrm{~S}$ & 96 & 1.0 & $255-256^{20}$ \\
\hline 45 & $4-\mathrm{CH}_{3} \mathrm{O}-\mathrm{C}_{6} \mathrm{H}_{4}$ & $\mathrm{H}$ & $\mathrm{CH}_{3}$ & $\mathrm{~S}$ & 80 & 4.5 & $179-180^{20}$ \\
\hline 46 & 4-HO- $\mathrm{C}_{6} \mathrm{H}_{4}$ & $\mathrm{H}$ & $\mathrm{CH}_{3}$ & $\mathrm{~S}$ & 75 & 4.5 & $245-246$ \\
\hline
\end{tabular}

$\bar{a}$ Isolated yield based on aldehyde while using $8 \mathrm{~mol} \%$ of catalyst. ${ }^{b}$ Compounds are known in the literature and were characterized by their physical and spectral data 
Table 2. Comparison of the yield of 5-ethoxycarbonyl-6-methyl-4-phenyl-3,4-dihydropyrimidin-2(1H)-one (1) synthesized by different catalysts

\begin{tabular}{|c|c|c|c|c|c|}
\hline Entry & Catalyst & Solvent $(\mathrm{mL})^{a}$ & Time (h) & Yield (\%) & Reference \\
\hline 1 & HTMA (Heulandite) & $\mathrm{AcOH}(25)$ & $4-5$ & 75 & 25 \\
\hline 2 & 12-Tungstophosphoric acid & $\mathrm{AcOH}(15)$ & $6-7$ & 75 & 27 \\
\hline 3 & $\mathrm{Sr}\left(\mathrm{NO}_{3}\right)_{2}$ & $\mathrm{AcOH}(37)$ & 6 & 78 & 32 \\
\hline 4 & 12-Molybdophosphoric acid & $\mathrm{AcOH}(4)$ & 5 & 80 & 33 \\
\hline 5 & Con. $\mathrm{H}_{2} \mathrm{SO}_{4}$ & EtOH (7) & 16 & 71 & $10,12,13$ \\
\hline 6 & $\mathrm{Y}\left(\mathrm{NO}_{3}\right)_{3} \cdot 6 \mathrm{H}_{2} \mathrm{O}$ & Solvent free & $20 \mathrm{~min}$ & 97 & $21 b$ \\
\hline 7 & $\mathrm{Y}(\mathrm{OAc})_{3} \cdot \mathrm{xH}_{2} \mathrm{O}$ & АсОН (3) & 4 & 92 & Present work \\
\hline
\end{tabular}

${ }^{a}$ The amount of solvent corresponding to $5 \mathrm{mmol}$ of aldehyde

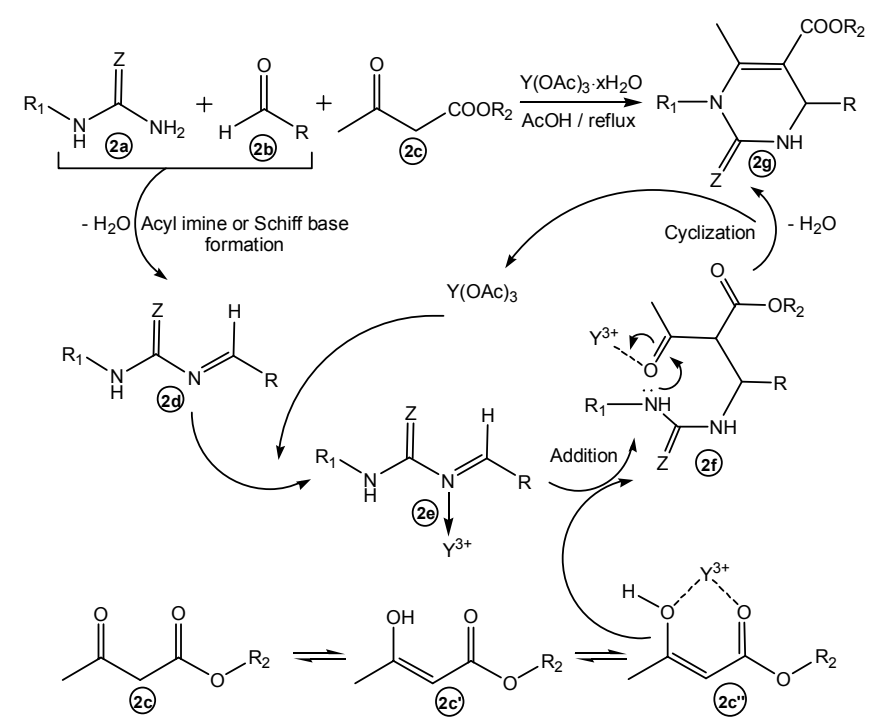

Scheme 2. Plausible mechanism for the formation of DHPM/DHPMthione

either electron-withdrawing or electron-donating substituents and achieved the corresponding DHPMs 1-13 (Table 1) in good to excellent yields (87 - 98\%). Besides urea, $N$-methylurea (DHPMs 15-20) and thiourea (DHPMs 21-26) were also employed successfully as substrate here without causing any significant decrease in yield (76 - 94\%). The derived DHPMs by the above said variants are of much interest with regard to their biological activity. ${ }^{4,5}$ Encouraged by the results obtained with ethyl acetoacetate, we examined the tolerance of another $\beta$-keto ester viz. methyl acetoacetate (DHPMs 27-33; 35-46) in this Biginelli reaction with varied aromatic aldehydes and urea or $N$-methylurea or thiourea and also accomplished better results ( 75 - 96\%). Meanwhile, even for aliphatic aldehyde, viz. butyraldehyde, which normally shows extremely poor yield in the Biginelli reaction with $\beta$-dicarbonyl compounds and urea, the corresponding dihydropyrimidin-2(1H)-ones $\mathbf{1 4}$ and $\mathbf{3 4}$ could be obtained in moderate yields (Table 1). Majority of the existing synthetic strategies have shown a great deal of variability in yield depending on the aromatic aldehyde and $N$-methylurea or thiourea used. However, our three-component reaction proceeded smoothly to furnish uniformly high yields, irrespective of the type of aromatic aldehyde carrying electron-rich or electron-poor substituents in either ortho or meta or para position, $\beta$-dicarbonyl compound and urea derivatives used. Therefore, this protocol tolerates many of the substitution patterns on all the three components to provide an array of dihydropyrimidinones as evidenced from Table 1.

Generally, all the reactions were very clean without any side products. Indeed, the crude products obtained are of high purity ( $>95 \%$ ascertained by ${ }^{1} \mathrm{H}$ NMR) and did not require any chromatographic separation (However, mild non-polar impurity encountered in few cases was easily removed by simple digestion in hexane for $15 \mathrm{~min}$ ). Table 2 describes the comparison of the present work towards the synthesis of DHPM 1 with other catalytic methods in literature $25,27,32,33,10-13,21 \mathrm{~b}$ which employ acetic acid or ethanol as medium or without any solvent. A close inspection of the Table 2 justifies the superiority of our method in terms of yield and relatively less solvent. Furthermore, the advantage of using acetic acid as medium is that it offers a homogeneous medium wherein the acetate function of yttrium acetate could create more positive charge over yttrium and thus allows the reactive species ( $\mathbf{2 d} \& \mathbf{2} \mathbf{c}$ - Scheme 2$)$ to be attracted towards the active sites ${ }^{34}$ which in turn reflects in formation of desired products in good yield. An interesting feature of this optimized protocol is that it enhances the yield compared to the classical Biginelli method and markedly decreases the reaction time from $18 \mathrm{~h}$ to $45 \mathrm{~min}-6.5 \mathrm{~h}$.

Mechanistically, the reaction proceeds through acid catalyzed formation of acylimine or Schiff's base (2d) from urea (2a) [or $\mathrm{N}$-methylurea or thiourea] and aldehyde (2b) (Scheme 2). Due to the presence of vacant $4 d$ orbital in the yttrium(III) ion, it stabilizes the then formed acylimine intermediate via coordination with yttrium(III) acetate (2e). This was followed by the addition to 1,3-dicarbonyl compound (2c), most likely through its yttrium (III) stabilized enolate form $\left(\mathbf{2} \mathbf{c}^{\prime \prime}\right)$, and produces an open chain ureide (2f) which upon subsequent cyclization and acid catalyzed dehydration furnished the desired target DHPM/DHPMthione (2g). ${ }^{14,35}$

\section{Conclusion}

In conclusion, we have developed a simple and high-yielding protocol for synthesizing a library of 3,4-dihydropyrimidin$2(1 H)$-(thio)ones by using yttrium(III) acetate hydrate as catalyst in a homogeneous medium. In addition to its simplicity and easy work up, the reaction condition is mild enough for the survival of a variety of sensitive functionalities such as $\mathrm{F}, \mathrm{Cl}, \mathrm{Br}$, 
$\mathrm{NO}_{2}, \mathrm{OH}, \mathrm{OMe}$ and $\mathrm{Me}$, which is lacking in some of the existing procedures. Purities and yields were not affected if $N$-methylurea or thiourea were used instead of urea. Therefore, we deem our protocol will find its way to furnish the needs of academia as well as pharmaceutical industries towards the synthesis of broad range of dihydropyrimidones.

Supporting Information. NMR spectra for all the compounds are furnished.

Acknowledgments. This work was supported by second stage of BK-21 program.

\section{References}

1. Schreiber, S. L. Science 2000, 287, 1964.

2. Kappe, C. O. Multicomponent Reactions; Zhu, J., Bienaymé, H., Eds.; Wiley-VCH: Weinheim, 2005; pp 95-120.

3. Bienaymé, H.; Hulme, C.; Oddon, G.; Schmitt, P. Chem. Eur. J. 2000, 6, 3321.

4. Kappe, C. O. Tetrahedron 1993, 49, 6937.

5. Kappe, C. O. Eur. J. Med. Chem. 2000, 35, 1043.

6. Ghorab, M. M.; Abdel-Gawad, S. M.; El-Gaby, M. S. A. Farmaco 2000, 55, 249.

7. Chikhale, R. V.; Bhole, R. P.; Khedekar, P. B.; Bhusari, K. P. Eur. J. Med. Chem. 2009, 44, 3645.

8. Mayer, T. U.; Kapoor, T. M.; Haggarty, S. J.; King, R. W.; Schreiber, S. L.; Mitchison, T. J. Science 1999, 286, 971.

9. Biginelli, P. Gazz. Chim. Ital. 1893, 23, 360.

10. Hu, E. H.; Sidler, D. R.; Dolling, U.-H. J. Org. Chem. 1998, 63, 3454.

11. Lu, J.; Bai, Y. Synthesis 2002, 466.

12. Folkers, K.; Harwood, H. J.; Johnson, T. B. J. Am. Chem. Soc. 1932, 54, 3751 .

13. Folkers, K.; Johnson, T. B. J. Am. Chem. Soc. 1933, 55, 2886.

14. Folkers, K.; Johnson, T. B. J. Am. Chem. Soc. 1933, 55, 3784.
15. Hassani, Z.; Islami, M. R.; Kalantari, M. Bioorg. Med. Chem. Lett. 2006, 16, 4479.

16. Salehi, P.; Dabiri, M.; Zolfigol, M. A.; Fard, L. A. B. Tetrahedron Lett. 2003, 44, 2889.

17. Fu, N. Y.; Yuan, Y. F.; Cao, Z.; Wang, S. W.; Wang, J. T.; Peppe, C. Tetrahedron 2002, 58, 4801.

18. Ananda Kumar, K.; Kasthuraiah, M.; Suresh Reddy, C.; Devendranath Reddy, C. Tetrahedron Lett. 2001, 42, 7873.

19. Adibi, H.; Samimi, K. A.; Beygzadeh, M. Catal. Commun. 2007, 8, 2119.

20. Ramalingan, C.; Kwak, Y. W. Tetrahedron 2008, 64, 5023.

21. (a) Sabitha, G.; Kiran Kumar Reddy, G. S.; Bhaskar Reddy, K.; Yadav, J. S. Tetrahedron Lett. 2003, 44, 6497. (b) Nandurkar, N. S.; Bhanushali, M. J.; Bhor, M. D.; Bhanage, B. M. J. Mol. Catal. A: Chem. 2007, 271, 14. (c) Ramalingam, S.; Kumar, P. Synth. Commun. 2009, 39, 1299.

22. Jin, T.; Zhang, S.; Li, T. Synth. Commun. 2002, 32, 1847.

23. Aridoss, G.; Amirthaganesan, S.; Ashok Kumar, N.; Kim, J. T.; Lim, K. T.; Kabilan, S.; Jeong, Y. T. Bioorg. Med. Chem. Lett. 2008, 18,6542 .

24. Aridoss, G.; Amirthaganesan, S.; Kim, M. S.; Kim, J. T.; Jeong, Y. T. Eur. J. Med. Chem. 2009, 44, 4199.

25. Tajbakhsh, M.; Mohajeranim, B.; Heravi, M. M.; Ahmadi, A. N. J. Mol. Catal. A: Chem. 2005, 236, 216.

26. Heravi, M. M.; Derikvand, F.; Bamoharram, F. F. J. Mol. Catal. A: Chem. 2005, 242, 173

27. Wannberg, J.; Dallinger, D.; Kappe, C. O.; Larhed, M. J. Comb. Chem. 2005, 7, 574.

28. Hegedüs, A.; Hell, Z.; Vígh, I. Synth. Commun. 2006, 36, 129.

29. Hojatollah, S.; Qing-Xiang, G. Chin. J. Chem. 2005, 23, 91.

30. Lewandowski, K.; Murer, P.; Svec, F.; Fréchet, J. M. J. J. Comb. Chem. 1999, 1, 105.

31. Singh, K.; Arora, D.; Singh, S. Tetrahedron Lett. 2006, 47, 4205.

32. Liu, C.; Wang, J.; Li, Y. J. Mol. Catal. A: Chem. 2006, 258, 367.

33. Heravi, M. M.; Bakhtiari, K.; Bamoharram, F. F. Catal. Commun. 2006, 7, 373.

34. Hsu, T. J.; Tan, C. S. Polymer 2001, 42, 5143.

35. Kappe, C. O. J. Org. Chem. 1997, 62, 7201. 